\title{
Prozess- und Anlagensicherheit
}

\author{
Michael Hohenberger und Harald Raupenstrauch
}

Lehrstuhl für Thermoprozesstechnik, Montanuniversität Leoben, Leoben, Österreich

Eingegangen 30. April 2020; angenommen 22. Mai 2020; online publiziert 17. Juni 2020

Zusammenfassung: Die Arbeitsgruppe Prozess- und Anlagensicherheit des Lehrstuhls für Thermoprozesstechnik an der Montanuniversität Leoben verfügt über umfangreiche Lehr- und Forschungskompetenzen. So wird beispielsweise zur Bestimmung sicherheitstechnischer Kenngrößen von Stäuben und Gasen ein eigens dafür ausgestattetes Labor betrieben. Die Forschungsschwerpunkte umfassen dabei die Untersuchung grundlegender Brand- und Explosionsmechanismen und den daraus folgenden Auswirkungen. Anwendung findet das Wissen in der Prävention von industriellen Katastrophen und Unfällen und deren Abwehr. Der Wissenstransfer erfolgt neben regulären Vorlesungen und Laborübungen durch einen berufsbegleitenden, postgradualen Universitätslehrgang in diesem Bereich.

Schlüsselwörter: Sicherheitstechnik, Anlagensicherheit, Staubexplosion, Notfall- und Katastrophenmanagement

\section{Process and Plant Safety}

Abstract: The working group Process and Plant Safety of the Chair of Thermal Processing Technology at the Montanuniversitaet Leoben has extensive expertise in safety related teaching and research activities. For example, a specially equipped laboratory is operated to determine safetyrelated parameters of dusts and gases. The research focuses on the investigation of fire and explosion mechanisms and the resulting effects. The knowledge is used in the prevention of industrial disasters and accidents as well as in the disaster response in industry and public. In addition to regular lectures and laboratory exercises, knowledge transfer also takes place in a part-time, postgraduate university course in the fields of process and plant safety, emergency and disaster management.

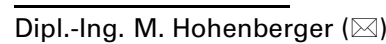

Lehrstuhl für Thermoprozesstechnik,

Montanuniversität Leoben,

Franz-Josef-Straße 18,

8700 Leoben, Österreich

michael.hohenberger@unileoben.ac.at
Keywords: Safety engineering, Plant safety, Dust explosion, Emergency and disaster management

\section{Einleitung}

Früher hauptsächlich im Bergbau und der chemischen Industrie bekannt, ist der Explosionsschutz längst in vielen industriellen Anwendungen von Bedeutung. Gegenwärtig stellt die Sicherheitstechnik für die Abfall- und Ressourcenwirtschaft eine große Herausforderung dar. Die Folge der Verwendung immer leistungsfähigeren Akkus (Li-lonen), welche häufig falsch entsorgt werden, und die separate Sammlung und Aufbereitung von Abfällen mit hohem Heizwert (hohes Verwertungspotential) sind schwer zu beurteilende und brandgefährliche Einsatzmaterialien. Speziell hier gilt es eine Bewertung potenziell gefährdeter Anlagen- und Lagerbereiche und die Dimensionierung von Schutzeinrichtungen vorzunehmen. Voraussetzung dafür sind aussagekräftige sicherheitstechnische Kenngrößen sowie das Verständnis zur Umsetzung.

Die Arbeitsgruppe Prozess- und Anlagensicherheit versteht es, durch theoretische und experimentelle Methoden diese Bewertungen fachgerecht umzusetzen. Ein umfangreich ausgestattetes sicherheitstechnisches Labor mit den entsprechenden Analysegeräten steht hierfür zur Verfügung. Zudem werden selbst entwickelte Prüf- bzw. Untersuchungsverfahren angewendet. Zur Konzeptionierung und laufenden Validierung der Experimente werden Simulationen der numerischen Strömungsmechanik (CFD), welche in Zusammenarbeit mit der Arbeitsgruppe Modellierung und Simulation des Lehrstuhls erfolgen, durchgeführt.

\section{Laborausstattung}

Seit Jahren werden am Lehrstuhl normgerechte Brennstoffanalysen und die Bestimmung von Parametern zum Brand- und Explosionsverhalten, sogenannte sicherheitstechnische Kenngrößen, vorgenommen. Eine 20-LiterApparatur ermöglicht die Messung folgender Kenngrö- 
Abb. 1: Ausstattung im sicherheitstechnischen Labor am Lehrstuhl für Thermoprozess technik (a) 20-Liter-Apparatur (b) Zündung eines Staub/ Luft-Gemisches in der MIKE 3-Apparatur
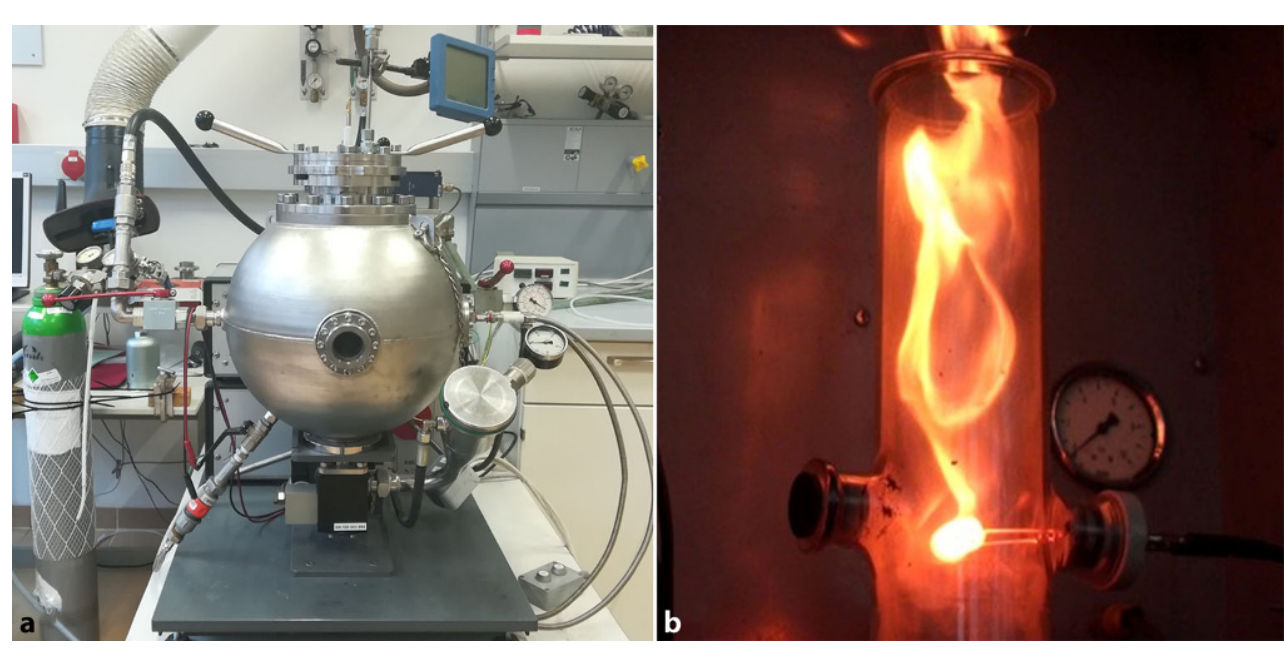

ßen von Staub/Luft-Gemischen, Gas/Luft-Gemischen und hybriden Gemischen:

- maximaler Explosionsdruck

- $\mathrm{K}_{\mathrm{ST}}$ - bzw. $\mathrm{K}_{\mathrm{G}}$-Wert

- Sauerstoffgrenzkonzentration

- Obere/untere Explosionsgrenzen

Für die normgerechte Bestimmung der Mindestzündenergie von brennbaren Staub/Luft-Gemischen steht eine MIKE 3-Apparatur zur Verfügung. Die Apparaturen sind in Abb. 1 dargestellt und werden in weiterer Folge näher beschrieben.

\subsection{0-Liter-Apparatur}

Der $\mathrm{K}_{\mathrm{ST}}-$ bzw. $\mathrm{K}_{\mathrm{G}}$-Wert ist ein staub- bzw. gas- und prüfverfahrensspezifischer Parameter, welcher die Grundlage für die Einteilung in Explosionsklassen bildet und ein Maß für Heftigkeit einer Explosion darstellt. Für die Bestimmung des Wertes wird der Explosionsüberdruck und die zeitliche

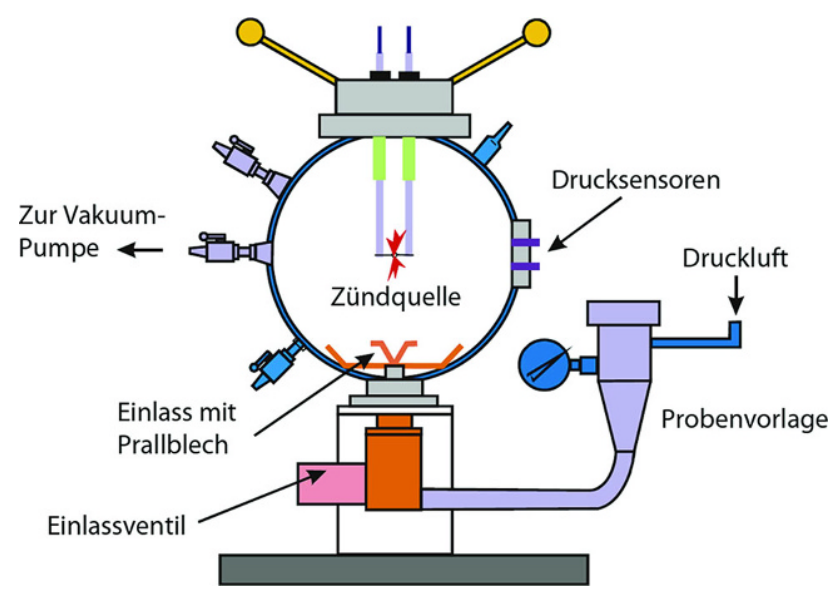

Abb. 2: Schematische Darstellung der 20-Liter-Apparatur [3]
Steigung des Druckanstiegs einer erfolgreich gezündeten Explosion gemessen.

Die 20-Liter-Apparatur besteht aus einer Prüfkammer, einer Hohlkugel aus rostfreiem Stahl, in der das brennbare Staub/Luft- oder Gas/Luft-Gemisch möglichst gleichmäßig verteilt und gezündet wird. Der Wassermantel, der die Prüfkammer umgibt, führt die Reaktionswärme der Explosion ab und ermöglicht die Einstellung unterschiedlicher Versuchstemperaturen. Die Apparatur ist in Abb. 2 schematisch dargestellt $[1,2]$.

\subsection{MIKE 3-Apparatur}

Die MIKE 3-Apparatur besteht im Wesentlichen aus einem modifizierten Hartmannrohr aus Glas (Abb. 3). Der zu untersuchende Staub wird am Boden lose aufgebracht und mittels eines Druckluftstoßes im Rohr verteilt. Zwei Elektroden im unteren Drittel der Apparatur dienen als Zündquelle. Zur Anwendung kommt die Prüfapparatur bei der Bestimmung der Mindestzündenergie (MZE). Das ist die niedrigste Energie, welche eine Hochspannungs-Kondensatorentladung aufbringen muss, um das zündwilligste Staub/LuftGemisch zur Zündung zu bringen [4].

Die Mindestzündenergie hilft bei der qualitativen Beurteilung von Gefahrensituationen in Betrieben. Häufig treten Staub/Luft-Gemische unter anderem auch bei erhöhten Temperaturen auf, wodurch sich die Mindestzündenergie erniedrigt. Aktuell wird am Lehrstuhl an einer Methode zur standardmäßigen Bestimmung der MZE von Staub/LuftGemischen unter diesen Bedingungen geforscht.

\section{Forschungstätigkeiten}

Die Tätigkeiten der Arbeitsgruppe erstreckt sich über alle Bereiche der Sicherheitstechnik, von Grundlagenforschung über Planung der Präventionsmaßnahmen bis hin zur Gefahrenabwehr. Einen Überblick über aktuelle Projekte wird in den folgenden Abschnitten gegeben. 


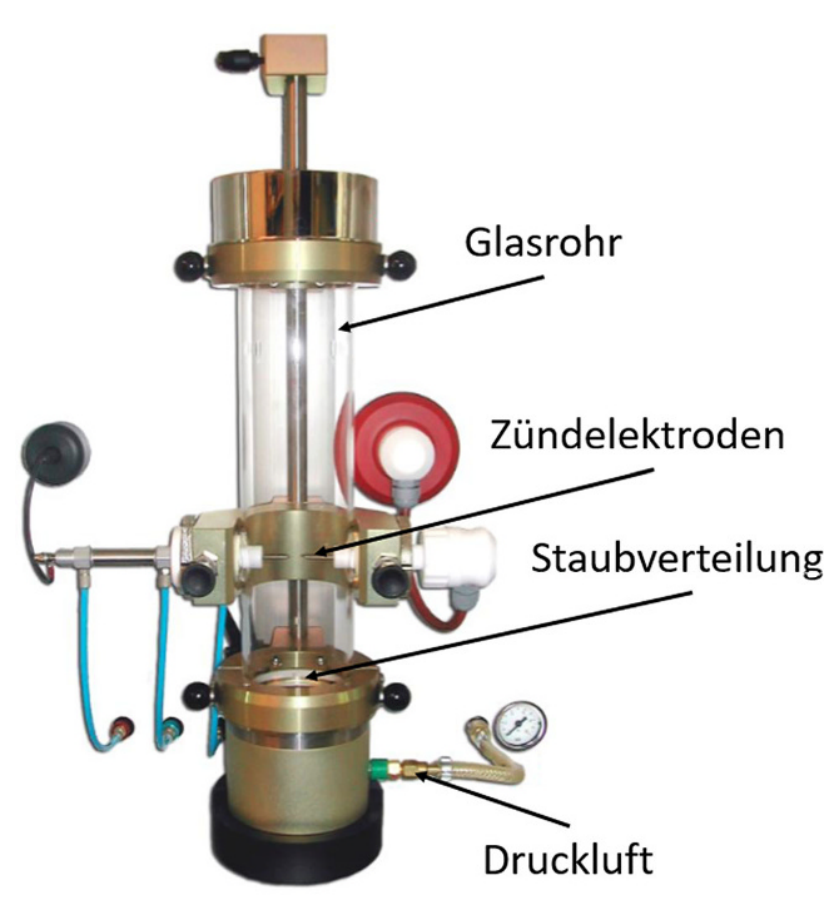

Abb. 3: Aufbau der MIKE 3-Apparatur

\subsection{Versuchstand nach der Tube-Methode}

In der Staubexplosionsforschung gibt es Bestrebungen, neben den sicherheitstechnischen Kenngrößen auch andere Kennparameter experimentell zu bestimmen und so sicherheitstechnische Einrichtungen, wie z. B. Berstscheiben, besser an den Produktionsprozess anpassen zu können. Besonderes Interesse besteht an der Übertragung dieser Parameter auf den industriellen Großmaßstab.

Unter Berücksichtigung dieses Aspekts wurde eine Großversuchsanlage nach der Tube-Methode auf Basis von CFD-Modellierungen konstruiert. Die Versuchsanlage besteht aus einem Stahlrohr mit einem Durchmesser von $800 \mathrm{~mm}$ und einer Höhe von $4 \mathrm{~m}$ (Abb. 4). Dabei wird der zu untersuchende Staub über vier Eintragsvorrichtungen am oberen Rohrende eingebracht, die Regelung der Fördermenge ist durch installierte Wägezellen gewährleistet. Gezündet wird das Staub/Luft-Gemisch mittels elektrischen Hochspannungs-Plasmabogen zwischen zwei Elektroden im Bodenteil. Ein eigens erstelltes Programm in LabVIEW regelt die Fördermenge und -zeit, löst die Zündung aus und erfasst die Messdaten.

Der Bau und die Testphase der Anlage konnten im Herbst 2019 erfolgreich abgeschlossen werden, die ersten Versuchsreihen werden voraussichtlich bis Herbst 2020 fertiggestellt. Durch die eigens entwickelte Messtechnik, bestehend aus Photodioden und Thermoelementen, kann die Geschwindigkeit der Flammenfront detektiert werden. An der Implementierung einer lasergestützten Staubkonzentrationsmessung wird derzeit gearbeitet. Ebenso sollen in Zukunft Forschungsfragen zu Staubexplosionen unter nichtatmosphärischer Umgebungsbedingungen erforscht werden.

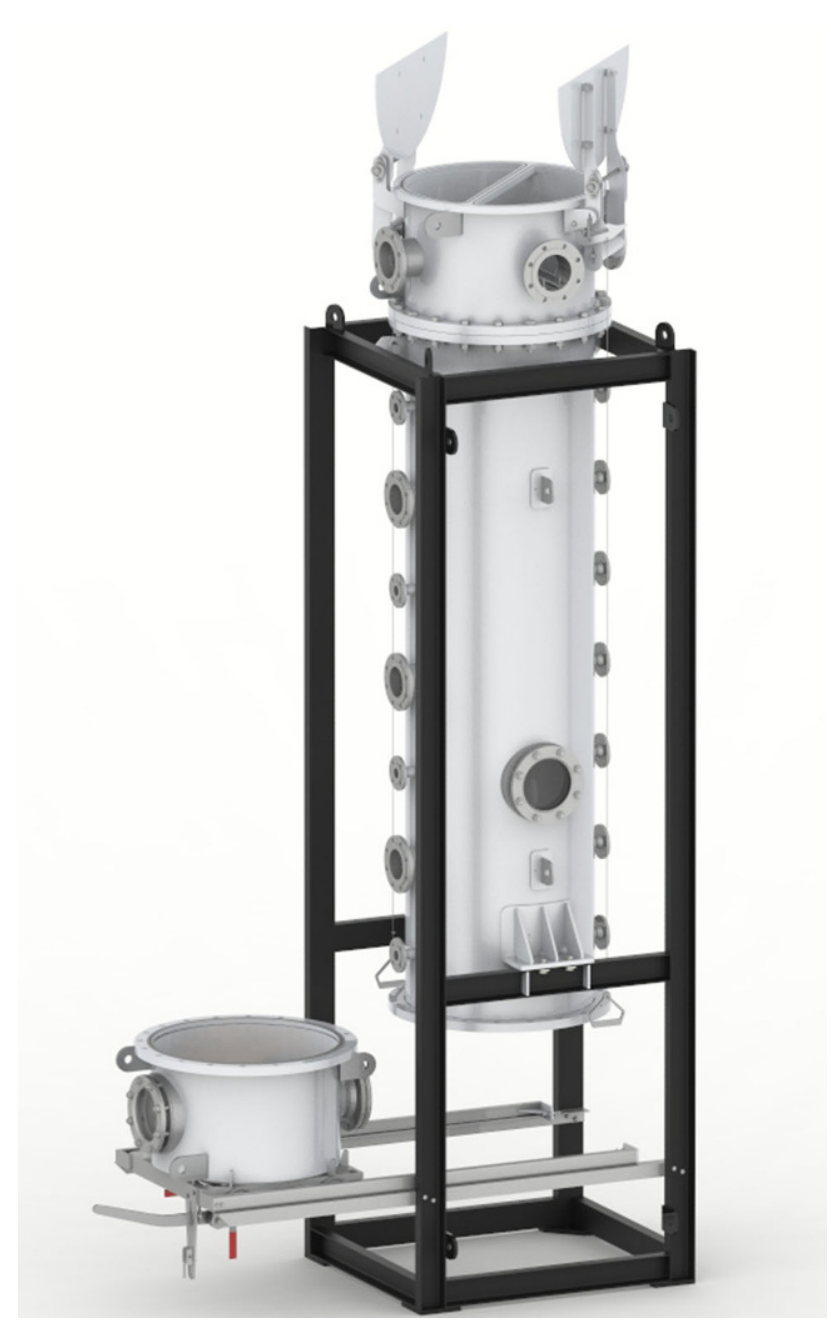

Abb. 4: Großversuchsanlage nach der Tube-Methode mit ausgefahrenem Bodenteil und geöffnetem Deckel

\subsection{Projekt „FIRETEST”}

In der modernen Brandbekämpfung haben Löschmittelzusätze zur Erhöhung der Löschwirkung von Wasser einen wichtigen Stellenwert. Einsatz finden diese Zusätze bei Wald-, Gebäude- oder Anlagebränden. Die Auswahl von geeigneten Zusätzen gestaltet sich insofern als schwierig, als Normen und Richtlinien, welche zur Beurteilung von Löschmittel angewendet werden, die chemischen und physikalischen Eigenschaften des Stoffgemisches beschreiben, nicht jedoch deren tatsächlichen Löschwirkung [5].

Hier setzt das innovative Prüfverfahren, welches im Rahmen des Projektes "FIRETEST" entwickelt wird, an. Ziel ist es, anhand einer neuen Prüfmethodik eine Klassifizierung unterschiedlicher Löschmittelzusätze nach ihrer Löschwirkung vorzunehmen. Vorversuche hinsichtlich eines Referenzbrandszenarios mit Kiefernholzstäben zeigten großes Potential (Abb. 5). Dabei konnte aufgrund der definierten Versuchsbedingungen die Löschzeit als Maß für die Effektivität des Löschmittels herangezogen werden. Gegenwärtig wird dieser Versuch in ein vollautomatisiertes Testverfahren umgebaut, um eine weitestgehende Unabhängig- 


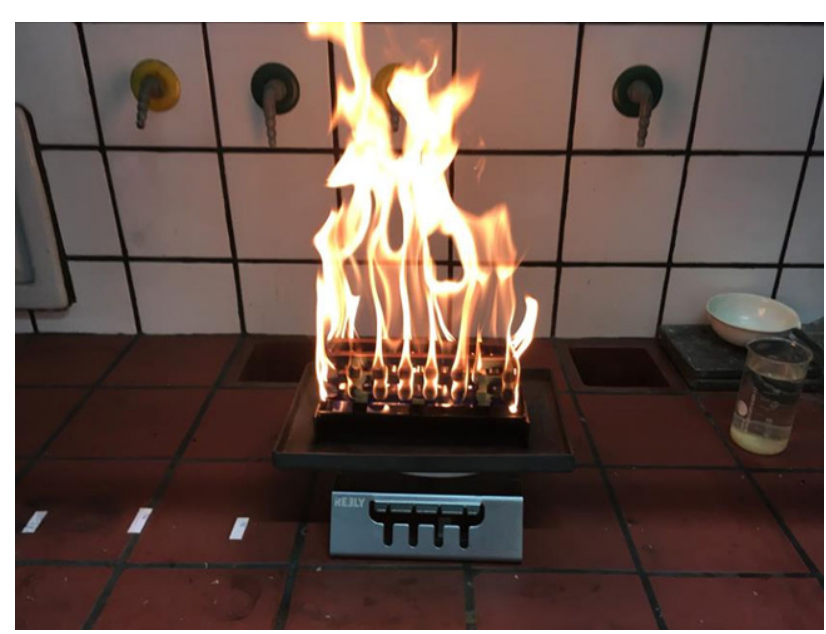

Abb. 5: Definierter Brandversuch im Labormaßstab [6]

keit von äußeren Beeinflussungen zu erreichen und einen gleichbleibenden Versuchsablauf zu schaffen.

Prüfverfahren und -abläufe für die Bewertung von Löschmittelzusätzen ermöglichen in Zukunft nicht nur eine Klassifizierung nach Wirksamkeit und infolgedessen eine für den Anwender nachvollziehbare Einstufung, es wird auch den Produktherstellern ermöglicht, eine gezielte Entwicklung durchzuführen.

\subsection{Projekt "AbER Innovation"}

In den letzten Jahren hat sich die Brandproblematik in der Abfallwirtschaft zunehmend verschärft. Gründe dafür sind nicht nur die eingangs bereits erwähnten steigenden Anteile an Lithiumionenbatterien, sondern auch kontinuierliche Veränderungen der Abfallzusammensetzung spielen eine Rolle. Als erschwerender Faktor kommt hinzu, dass die Ursachen für Brände sehr vielfältig sind: Selbstentzündung, biologische und chemische Reaktionen, heiß laufende Maschinenteile, elektrische Einwirkungen, Umwelteinflüsse und Brandstiftung sind nur einige Beispiele, die tatsächliche Brandursache bleibt in vielen Fällen jedoch unbekannt $[7,8]$.
Im Mittelpunkt des Projektes „AbER Innovation“ steht der Aufbau und Austausch von Know-how in einem Netzwerk zu dieser aktuellen Thematik. Einerseits wird das Know-how durch Forschung und intensiven interdisziplinären Wissensaustausch gesteigert. Andererseits werden die hier geteilten Erfahrungen und gewonnenen Erkenntnisse in einer dynamischen und erweiterbaren Austauschplattform für die Stakeholder bereitgestellt.

Die Arbeitsgruppe Prozess- und Anlagensicherheit wird hierfür innovative Experimente zur Beschreibung der Brandkinetik und dem Brandgasverhalten von Abfällen im Labor- und Realmaßstab abwickeln. Angewendet wird ein Differenzialkreislaufreaktor, mit dem es möglich ist, die Bildungsraten von Brandgasen organischer Stoffe im niederen Temperaturbereich abzubilden. Zudem besteht die Möglichkeit, Messungen von Brandgasen und Temperaturen in Lagerbereichen durchzuführen. Die entsprechende Messtechnik befindet sich in der Entwicklungsphase. Mit der Kenntnis dieser Größen und weiterführender CFDSimulationen (Abb. 6) sollen die Bedingungen in Anlagen und Haufwerken genauer beschrieben werden, um so Methoden der Brandvermeidung, -früherkennung und -löschung zielgerechter einsetzen zu können.

\subsection{Projekt „ERIMAPS”}

CBRNe-Substanzen zählen zu den chemisch, biologisch, radioaktiven, nuklearen und explosiven Gefahrenstoffen, früher auch ABC-Gefahrenstoffe genannt. Die Freisetzung dieser Substanzen führt zu großen Herausforderungen für die Einsatzorganisationen. In Zusammenarbeit mit ebenjenen Einsatzkräften verfolgt das Projekt ERIMAPS das Ziel, die anfänglichen Entscheidungsträger bestmöglich zu unterstützen. Denn vor allem in urbanen Räumen reicht es nicht aus, lediglich Gefährdungsradien, wie es aktuell der Fall ist, zu ermitteln. In die Entscheidung müssen auch Gefährdungen von Personen und kritischen Objekten (z.B. Kindergärten, Einkaufszentren, Wohngebäude, etc.) miteinfließen.

Um die Lage am Einsatzort schnell und zuverlässig beurteilen zu können, kommt ein System von Daten und Rechenmodellen zur Anwendung, die miteinander verknüpft
Abb. 6: CFD-Simulation eines Lagerbereiches

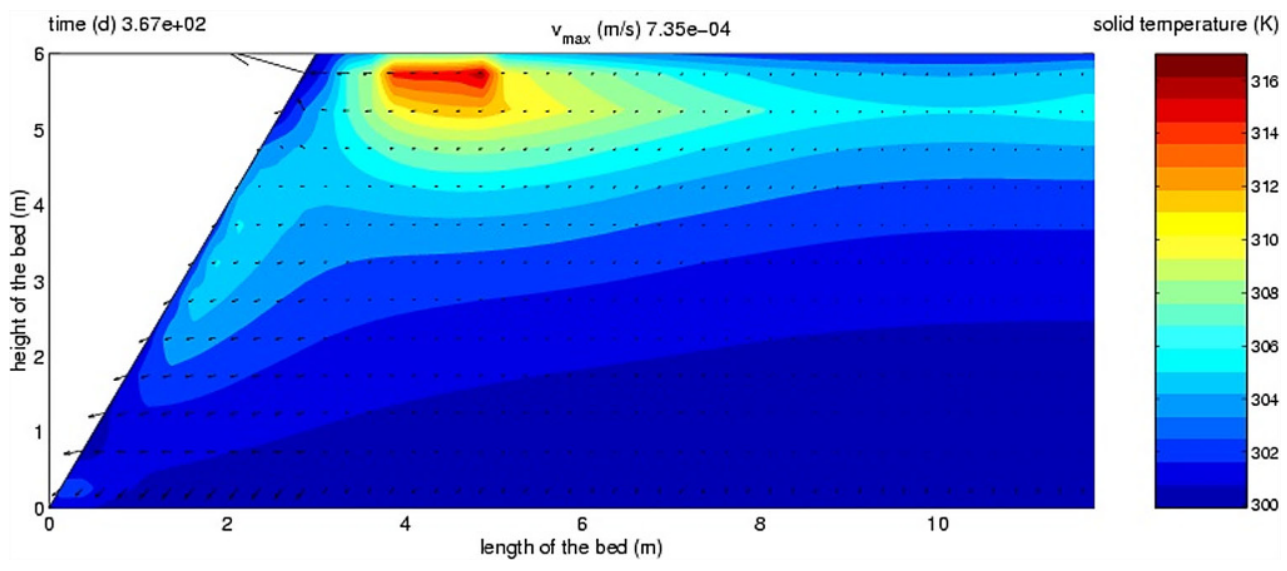


werden. Modelle der Dispersion und atmosphärischer Daten erlauben die Abschätzung der Stoffausbreitung. Mit der Modellierung von Effekten auf Gebäude und Personen und die anschließende Implementierung von Infrastrukturdaten und Daten über Personenströme lassen sich hochaktuelle Risikokarten inklusive Gefahrenzoneneinteilungen erstellen.

Dieses lokale „Risk-Mapping“ wird die zukünftige Herangehensweise der Einsatzorganisationen bei CBRNe-Gefahren wesentlich beeinflussen. Die Folge von schnellen, zielgerichteten und aktuellen Einsatzdaten ist die maßgebliche Effizienzsteigerung im Ressourcenmanagement, unter anderem der Einteilung von Personen und Geräte.

\section{Universitätslehrgang für Katastrophen- management}

Die Corona-Krise hat verdeutlicht, wie schnell eine Veränderung des Alltags stattfinden kann. Dabei können die grundlegenden Bedürfnisse nach Orientierung und Sicherheit nur durch ein exzellentes Krisen- und Katastrophenmanagement sichergestellt werden. Eine koordinierte Risikovorsorge und die erfolgreiche Bewältigung der Herausforderungen setzt Schlüsselkräfte voraus, die strategische Positionen in der öffentlichen Verwaltung und in Unternehmen besetzen.

Gemeinsam mit dem Lehrstuhl für Wirtschaft- und Betriebswissenschaften bietet der Lehrstuhl für Thermoprozesstechnik einen jährlich stattfindenden, berufsbegleitenden postgradualen Universitätslehrgang im Bereich Prozess- und Anlagensicherheit, Notfall- und Katastrophenmanagement an. Dieser ist fokussiert auf die Ausbildung von Schlüsselkräften und umfasst die Fachbereiche Risiko- und Einsatzmanagement, Wissenschaft und Führung für technische Notfälle und Naturkatastrophen. Im neu entwickelten Curriculum wird insbesondere auf interdisziplinäre Kompetenzentwicklung gesetzt. Nur durch fachübergreifende Zusammenarbeit können aktuelle gesellschaftliche Herausforderungen innovativ gelöst werden. Die Lehrinhalte werden dabei forschungsgeleitet und praxisrelevant vermittelt. Nähere Informationen gibt es auf https://ulg-pank.at/.

\section{Zusammenfassung}

Die Erforschung von Brand- und Explosionsmechanismen ermöglicht die Planung und Berechnung von Präventionsund Abwehrmaßnahmen für Industrie und Öffentlichkeit. Der Lehrstuhl für Thermoprozesstechnik besitzt auf diesem Gebiet Fähigkeiten, welche von Grundlagenforschung über praktische Untersuchungen im Labor- und Realmaßstab bis hin zu mathematischen Modellen und Simulationskompetenzen reichen. Der Lehrstuhl ist - auch durch die Kooperation mit wissenschaftlichen und industriellen Partnern sowie Einsatzkräften - ein kompetenter Partner für eine Vielzahl von sicherheitstechnischen Fragestellungen. Das Portfolio wird durch den Universitätslehrgang speziell für Entscheidungsträger in Industrie und öffentlichem Dienst abgerundet.

Funding. Open access funding provided by Montanuniversität Leoben.

Open Access Dieser Artikel wird unter der Creative Commons Namensnennung 4.0 International Lizenz veröffentlicht, welche die Nutzung, Vervielfältigung, Bearbeitung, Verbreitung und Wiedergabe in jeglichem Medium und Format erlaubt, sofern Sie den/die ursprünglichen Autor(en) und die Quelle ordnungsgemäß nennen, einen Link zur Creative Commons Lizenz beifügen und angeben, ob Änderungen vorgenommen wurden.

Die in diesem Artikel enthaltenen Bilder und sonstiges Drittmaterial unterliegen ebenfalls der genannten Creative Commons Lizenz, sofern sich aus der Abbildungslegende nichts anderes ergibt. Sofern das betreffende Material nicht unter der genannten Creative Commons Lizenz steht und die betreffende Handlung nicht nach gesetzlichen Vorschriften erlaubt ist, ist für die oben aufgeführten Weiterverwendungen des Materials die Einwilligung des jeweiligen Rechteinhabers einzuholen.

Weitere Details zur Lizenz entnehmen Sie bitte der Lizenzinformation auf http://creativecommons.org/licenses/by/4.0/deed.de.

\section{Literatur}

1. ÖNORM 14034-1: Bestimmung der Explosionskenngröße von Staub/Luft-Gemischen Teil 1: Bestimmung des maximalen Explosionsdruckes pmax von Staub/Luft-Gemischen (01.06.2005)

2. Cesana, Ch.; Siwek, R.: Handbuch der 20-I-Apparatur 7.1, http:// cesana-ag.ch/download/B000_070.pdf (07.10.2019)

3. Denkevits, A.: Dust explosion experiments measurements of explosion indices of graphite dust in hydrogen-containing atmospheres, Forschungsbericht, Forschungszentrum Karlsruhe, 2005

4. Cesana, Ch.; Siwek, R.: Handbuch zu MIKE 3.5, http://cesana-ag.ch/ download/B021_070.pdf (07.10.2019)

5. National Fire Protection Association: Standard on Wetting Agents, U.S. Standard NFPA 18, 2017

6. Holzer, A.: Löschversuche im Labormaßstab, Projektarbeit, Leoben, Montanuniversität, Lehrstuhl für Thermoprozesstechnik, 2017

7. Nigl, T.; Pomberger R.: Brandgefährliche Abfälle - Über Risiken und Strategien in der Abfallwirtschaft, Konferenzbeitrag zur Recy\&DepoTech 2018, Leoben, Montanuniv., 2018

8. Nigl, T.; Rübenbauer W.: Quantitative Ursachenforschung zu Brandfällen in der österreichischen Abfallwirtschaft, BAT-Safe - Projektinterner Bericht zu Arbeitspaket 1.1, Montanuniv. Leoben, 2018

Hinweis des Verlags. Der Verlag bleibt in Hinblick auf geografische Zuordnungen und Gebietsbezeichnungen in veröffentlichten Karten und Institutsadressen neutral. 